PRePAREd For SUbMission to JINST

$14^{\text {Th }}$ Topical Seminar on Innovative Particle and Radiation Detectors

3.-6. OCTOBER 2016

SiEna, ITALY

\title{
The Phase II ATLAS Pixel Upgrade: The Inner Tracker (ITk)
}

\author{
Tobias Flick on behalf of the ATLAS collaboration \\ University of Wuppertal, \\ Gaußstraße 20, 42097 Wuppertal, Germany \\ E-mail: flick@uni-wuppertal.de
}

\begin{abstract}
AвSTRACT: The entire tracking system of the ATLAS experiment will be replaced during the LHC Phase II shutdown (foreseen to take place around 2025) by an all-silicon detector called the ITk (Inner Tracker). The pixel detector will comprise the five innermost layers, and will be instrumented with new sensor and readout electronics technologies to improve the tracking performance and cope with the HL-LHC environment, which will be severe in terms of occupancy and radiation. The total surface area of silicon in the new pixel system could measure up to $14 \mathrm{~m}^{2}$, depending on the final layout, which is expected to be chosen in early 2017. Four layout options are being investigated at the moment, two with forward coverage to $|\eta| \leq 3.2$ and two to $|\eta| \leq 4$. For each coverage option, a layout with long barrel staves and a layout with novel inclined support structures in the barrel-endcap overlap region are considered. All potential layouts include modules mounted on ring-shaped supports in the endcap regions. Supporting structures will be based on low mass, highly stable and highly thermally conductive carbon-based materials cooled by evaporative carbon dioxide. Different designs of planar, 3D, and CMOS sensors are being investigated to identify the optimal technology for the different pixel layers. In parallel, sensor-chip interconnection options are being evaluated in collaboration with industrial partners to identify reliable technologies when employing very thin $(100 \mu \mathrm{m}$ to $150 \mu \mathrm{m})$ chips. While the RD53 Collaboration is developing the new readout chip, the pixel off-detector readout electronics will be implemented in the framework of the general ATLAS trigger and DAQ system. A readout speed of up to $5 \mathrm{Gbit} / \mathrm{s}$ per data link (FE-chip) will be needed in the innermost layers going down to $640 \mathrm{Mbit} / \mathrm{s}$ for the outermost. Because of the very high radiation level inside the detector, the first part of the transmission has to be implemented electrically, with signals converted for optical transmission at larger radii. Extensive tests are being carried out to prove the feasibility of implementing serial powering, which has been chosen as the baseline for the ITK pixel system due to the reduced material in the servicing cables foreseen for this option.
\end{abstract}

KeYwords: Particle tracking detectors, Hybrid detectors, Radiation-hard detectors 


\section{Contents}

1 Introduction 1

2 ITk Layout 2

3 ITk Pixel Modules $\quad 2$

3.1 Sensors 3

3.1.1 Planar Sensors 3

3.1.2 3D Sensors 4

3.2 Front-end Electronics 5

3.3 Monolithic Sensors 6

4 Powering and Detector Control 6

4.1 Serial Powering 6

4.2 Detector Control 6

4.2.1 The DCS Chip and Controller 7

5 Detector Readout Architecture $\quad 8$

6 Conclusion $\quad 8$

\section{Introduction}

In 2024-2026 the Large Hadron Collider (LHC) will be upgraded to the High Luminosity-LHC (HL-LHC). HL-LHC will operate at a five times higher luminosity $\left(5-7 \times 10^{34} \mathrm{~cm}^{-2} \mathrm{~s}^{-1}\right)$. As a consequence, the detectors will have to handle higher particle densities leading to higher occupancies in the detector components and higher radiation levels. The number of events per collision will increase from around 25 to up to 140-200 (depending on the luminosity) events per collision. As the tracking systems of the detectors at HL-LHC have to cope with this increased particle multiplicity, the ATLAS detector [1] will upgrade its inner detector during Phase II (2024-26) completely. The currently existing inner detector, providing the track information of crossing particles, will be replaced by an all-silicon tracking system consisting of a 5-layer Pixel detector as the innner part and a 4-layer Strip detector as the outer part. All components of the new Inner Tracker (ITk) are currently under development. In the next sections an overview of this R\&D program for most of the different components needed for the ITk Pixel system is described. 

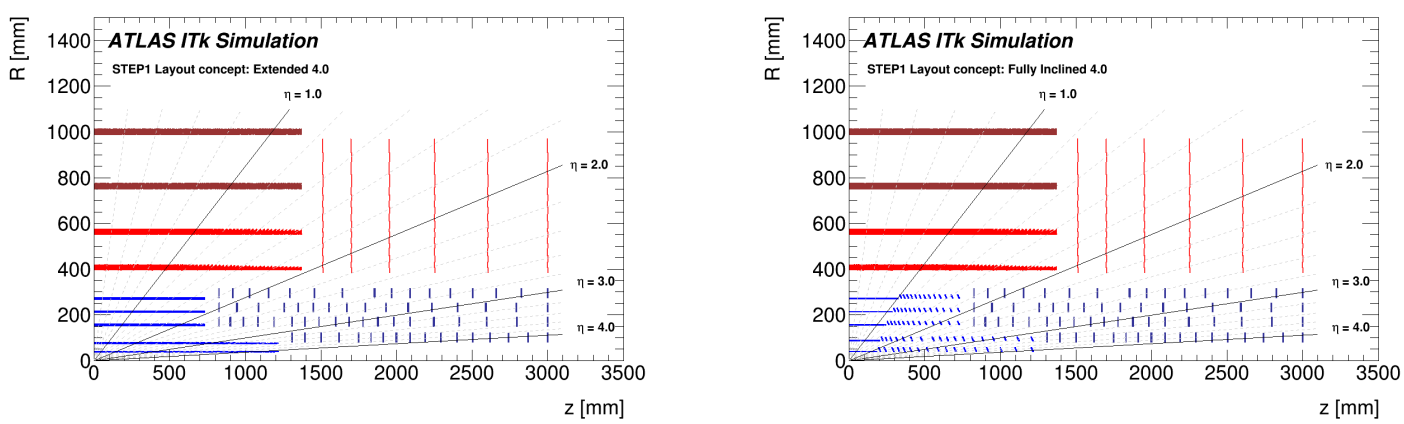

Figure 1. Simulated hit positions on the R-z plane for an ATLAS Inner Tracker layout with extended inner barrel layers (left) and inclined inner and outer barrel layer (right), optimized for measuring at least nine space points on tracks up to $|\eta| \leq 4.0$ for $\left|z_{0}\right|<150 \mathrm{~mm}$. Pixels (blue), short strips (light red) and long strips (dark red) are shown. [2].

\section{ITk Layout}

The final layout of the ITk Pixel detector is not yet decided. Two important decisions are pending: forward angle coverage ( $\eta \leq 3.2$ or $\eta \leq 4.0)$ and use of flat or inclined structures in the barrel layers. While in the flat layout the modules of the barrel section would be located parallel to the beam axis, in the inclined layout the outer modules of a stave would be inclined and therefore tracks originating from the interaction point would cross the modules at an angle close to normal. The latter option can potentially reduce the number of modules needed. Ongoing simulation studies will show the best option which then will become the baseline layout for the ITk pixel system. Figure 1 shows the two different options for angle coverage of $\eta \leq 4.0$.

\section{ITk Pixel Modules}

The basic electrical unit of the Pixel detector is a module. Depending on the finally chosen layout about 10,000 modules are needed for ITk. The baseline module concept is a hybrid module which uses a sensor and a readout chip bump bonded to each other at the pixel level. In addition, other concepts are being investigated like monolithic CMOS Pixel detectors especially for the outer layers (see section 3.3).

The type of modules will depend on the layer, the layout option (inclined or flat), and on the type of sensor to be used. 3D sensors (see section 3.1.2) will result in single chip modules, while planar sensors (see section 3.1.1) can be built in $2 \times 2$ or $1 \times 2$ chip modules. The inclined part of a stave would be equipped with $1 \times 2$ chip modules, while for the flat part for the outer layers and for the disks $2 \times 2$ chip modules will be used.

Because the new pixel readout chip is still in development the final size of this chip is not yet defined, its dimensions are assumed to be similar to the FE-I4 pixel chip $\left(\sim 17 \times 20 \mathrm{~mm}^{2}\right.$ for the active region and a periphery region of $\sim 2 \times 20 \mathrm{~mm}^{2}$ ). Accordingly, quad-chip modules would have dimensions of around $4 \times 4 \mathrm{~cm}^{2}$ and double chip modules around $2 \times 4 \mathrm{~cm}^{2}$.

3D sensor modules would be preferably used for the innermost layers, because of their advantages in power consumption and radiation tolerance. Planar sensors can be used for double-chip 
and quad-chip modules due to the large size sensor production feasibility.

To avoid inactive regions in between the chips longer pixels are foreseen in the sensor design as well as minimising the edge region while still preventing the voltage break down. The technique to be used depends on the sensor and module type choice. Chips and sensors will be as thin as possible. Handling and production yield (favouring thicker devices) have to be balanced against material reduction and radiation tolerance (favouring thinner devices). Finally, a flexible printed circuit to which the needed passive components are assembled is glued to the module and provides all connections to and within the module. The connections between the module and this printed circuit are done with standard wire bonds.

All chips within the module will be controlled in parallel (shared clock and command inputs), but the module may have one or several dedicated data outputs. In order to keep the amount of data cables as low as possible, data lines of read-out chips might be combined into a higher bandwidth signal wherever possible.

\subsection{Sensors}

Different sensor technologies are being investigated with several vendors for each technology, a new generation of planar sensors as well as 3D sensors, which build the basis for the well known hybrid module composition. The final pixel size is still under study. For the reduced pixel cell area of $250 \mu \mathrm{m}^{2}$ two sets of dimensions are being investigated: $25 \times 100 \mu \mathrm{m}^{2}$ and $50 \times 50 \mu \mathrm{m}^{2}$. Pixel cell designs have been implemented for the different sensor technologies and are under investigation now. Once the final layout has been chosen these two options need to be compared concerning tracking performance to give an indication which option to choose. The final read-out chip providing the smaller pixel pitch will also be essential to study real assemblies with the different pixel geometries.

\subsubsection{Planar Sensors}

A new generation of planar pixel sensors using n-in-p technology (current sensor are built in n-in-n technology) is under development for ITk. The final thickness of the devices has to be defined still. Currently, investigations for thin devices, $100 \mu \mathrm{m}$ to $150 \mu \mathrm{m}$, are ongoing. The aim is to provide a sensor which is thinner compared to the sensors used in IBL $(200 \mu \mathrm{m})$ or the outer layers of the current Pixel detector $(250 \mu \mathrm{m})$, which nevertheless provides a sufficient hit signal to be read out by the FE-chip. An advantage of the n-in-p technology is single side processing and reduced handling complexity. The guard ring structure is implemented on the front side, leaving the edges of the sensor at a potential close to the one of the backside. To avoid sparking between the sensor periphery and the FE-chip, isolation techniques have been investigated successfully. Either deposition of Benzocyclobutene (BCB) or parylene would be possible [3].

The cell structure design has been optimised with respect to the smaller pitch of the ITk readout chip based on beam test results obtained with FE-I4 chips. Preliminary electrical characterisation of these devices can be found in Ref. [4].

Thin planar sensors have been irradiated up to a fluence of $10^{16} \mathrm{n}_{\mathrm{eq}} / \mathrm{cm}^{2}$ to investigate the feasibility of employing them in the innermost pixel layers. A hit efficiency up to $97 \%$ with FE-I4 modules has been reached in test-beam measurements by $100 \mu \mathrm{m}$ thin sensors at a bias voltage of $350 \mathrm{~V}$ for a fluence of $5 \times 10^{15} \mathrm{n}_{\mathrm{eq}} / \mathrm{cm}^{2}$. Comparing this performance with those of thicker sensors it can be stated that $100 \mu \mathrm{m}$ thin sensors can provide the same tracking efficiency at lower 

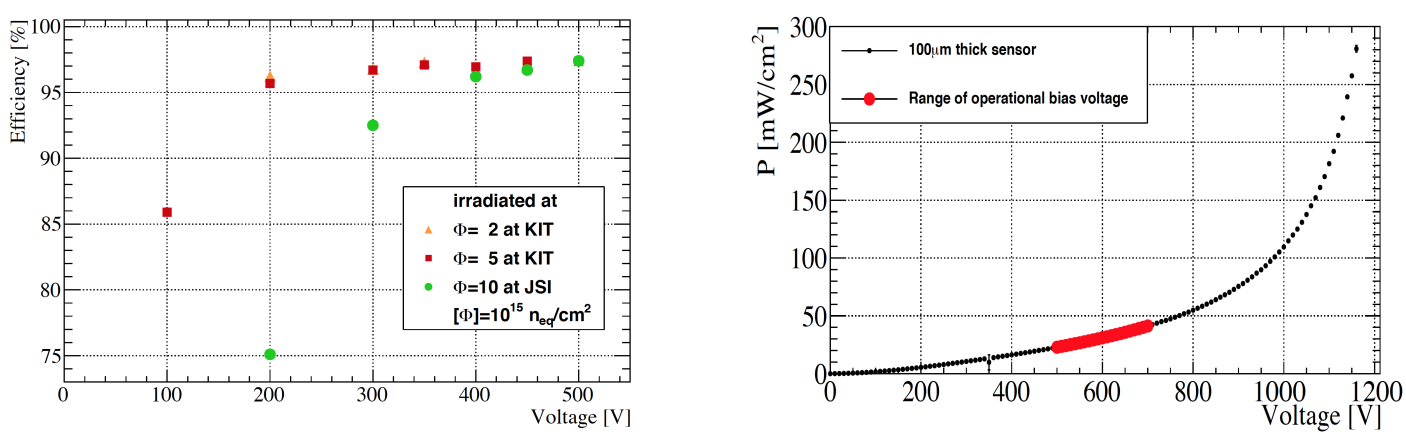

Figure 2. Left: Comparison of hit efficiencies of FE-I4 modules with $100 \mu \mathrm{m}$ thin planar sensors irradiated from a fluence of $2 \times 10^{15} \mathrm{n}_{\mathrm{eq}} / \mathrm{cm}^{2}$ to $10^{16} \mathrm{n}_{\mathrm{eq}} / \mathrm{cm}^{2}$. Right: Power dissipation estimated for $100 \mu \mathrm{m}$ thin sensors at a temperature of $-25^{\circ} \mathrm{C}$ irradiated to a fluence of $10^{16} \mathrm{n}_{\mathrm{eq}} / \mathrm{cm}^{2}$, where the range of possible operational bias voltages is based on the results shown in the left plot. The values of the leakage currents used in the calculation are for a sensor after 11 days of annealing at room temperature [5].

bias voltages. At a fluence of $10^{16} \mathrm{n}_{\mathrm{eq}} / \mathrm{cm}^{2} 500 \mathrm{~V}$ are needed to reach a hit efficiency of $97 \%$ in test-beam measurements. Based on this result, the power dissipation has been estimated to be in the range of $25 \mathrm{~mW} / \mathrm{cm}^{2}$ to $45 \mathrm{~mW} / \mathrm{cm}^{2}$ at a temperature of $-25^{\circ} \mathrm{C}$ for a sensor after 11 days of annealing (see Fig. 2).

\subsubsection{D Sensors}

3D silicon detectors [6] are candidates to be used for the innermost layer(s) of the barrel pixel system and some of the inner end-cap rings due to their excellent radiation hardness at low operational voltages as well as moderate temperatures with low power dissipation compared to planar sensors. 3D silicon detectors have recently undergone a rapid development from $R \& D$ to industrialisation with their first operation in the IBL.

IBL-generation 3D pixel detectors coupled to FE-I4 pixel electronics have been found to have hit efficiencies in testbeam measurements larger than $97 \%$ at $170 \mathrm{~V}$ after irradiation to $10^{16} \mathrm{n}_{\text {eq }} / \mathrm{cm}^{2}$

$50 \times 50 \mu \mathrm{m}^{2}, 1 \mathrm{E}$

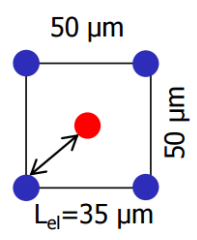

$25 \times 100 \mu \mathrm{m}^{2}, 2 E$



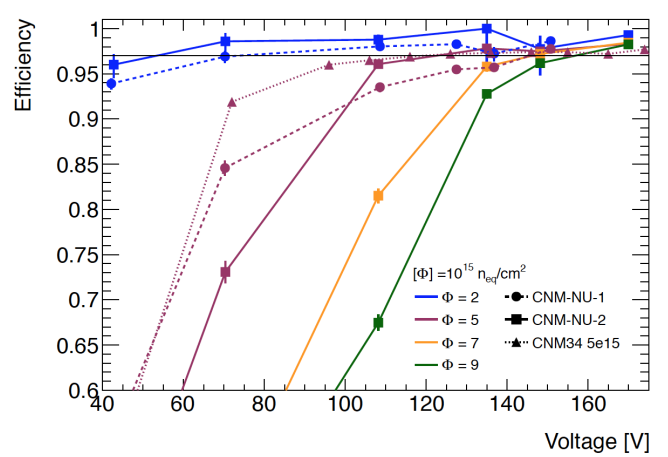

Figure 3. Left: Design of $3 \mathrm{D}$ pixel cells with $50 \times 50 \mu \mathrm{m}^{2}$ and $25 \times 100 \mu^{2}$ size [6]. Right: Hit efficiency vs. voltage for different fluences in various 3D sensor devices. The $97 \%$ benchmark efficiency is marked. [6]. 
for normally incident minimum ionizing particles with a power dissipation of $15 \mathrm{~mW} / \mathrm{cm}^{2}$ at a temperature of $-25^{\circ} \mathrm{C}$ [6], see 3 . The $3 \mathrm{D}$ design under development for the ITk detector builds on the successful IBL 3D sensor. Columns of $5 \mu \mathrm{m}$ to $10 \mu \mathrm{m}$ diameter are alternately $\mathrm{n}$ - and p-type doped into the high resistivity p-type silicon bulk, defining the pixel configuration. The 3D sensor technology inherently allows for slim edges of $15 \mu \mathrm{m}$ to $150 \mu \mathrm{m}$ [6], or even active edges sensitive up to the physical sensor edge [7]. Productions of 3D devices with smaller pixel sizes of $25 \times 100$ and $50 \times 50 \mu \mathrm{m}^{2}$ compatible with the FE-I4 chip have already been carried out and the prototypes are currently being tested. Furthermore, specific productions of 3D sensors compatible with the RD53 chip have also been completed.

\subsection{Front-end Electronics}

The new front-end ASIC is currently under development. The RD53 Collaboration [8] is developing a pixel readout chip for both ATLAS and CMS usage at the HL-LHC. The chip must cope with the HL-LHC requirements in terms of hit occupancy and radiation levels. In ITk the chip will be used in all layers. Nevertheless, the most critical performance is driven by the innermost layer. Compared to the IBL pixel chip the new chip will be improved in sevaral aspects. The pixel size will be reduced by a factor of five. The hit threshold will be around 600 electrons (compared to 2000 electrons) and triggers at a rate of $1 \mathrm{MHz}$ can be handled. The transmission bandwidth is increased to $5 \mathrm{Gbit} \mathrm{s}^{-1}$ (compared to $160 \mathrm{Mbit} \mathrm{s}^{-1}$ ). All this has been implemented without a significant increase of the power consumption of the chip. The ASIC is being designed in $65 \mathrm{~nm}$ CMOS technology (through a CERN frame contract).

The small feature size is critical to create the small pixel cells being needed for ITk. Radiation studies of $65 \mathrm{~nm}$ feature size process revealed an unexpected issue, the previously unknown effect called Radiation Induced Short Channel Effect (RISCE). Together with the already known Radiation Induced Narrow Channel Effect (RINCE), it is a CMOS damage mechanism, which leads to the fact, that small transistors, which are needed for high logic density designs, are bad for radiation tolerance [9]. Therefore, modest compromises have to be made on logic density. Taking this into account the RD53 chip still provides much higher logic density than the FE-I4 and can be qualified up to a dose of 5 MGy. Nevertheless, the innermost two layers have to be built removable to provide functionality over the full planned operation time of HL-LHC.

A first prototype ASIC (FE65-P2) [10] has been produced and is currently under test. FE65-P2 has the same pixel size and same CMOS process as the foreseen prototype chip RD53-A (submission in spring 2017). FE65-P2 also demonstrates the performance of the analog front-end part to be used in RD53-A and validates the superb analog-digital isolation needed for 600 electron threshold stable operation. The discriminator threshold of a bare FE65-P2 was tuned to values below 600 electrons. While a significant temperature variation of the threshold is observed on irradiated chips, pixels with a 'radiation hard' front-end variant show the smallest effect, at a level acceptable for operation. Generally, performance is still good even after $5 \mathrm{MGy}$. The threshold to detect a charge pulse within $25 \mathrm{~ns}$ was measured to be below 500 electrons for a discriminator threshold of 300 electrons. FE65-P2 bump bonded to sensors show a small increase in noise with nominal noise achieved at a bias voltage of $-10 \mathrm{~V}$ (full depletion expected at $-300 \mathrm{~V}$ ) which confirms expectations that the noise performance is most sensitive to the inter-pixel capacitance. Data were successfully taken with a $50 \mathrm{MeV}$ proton beam which shows pixel hit clusters the size of which matches the 
expected depletion depth. These excellent results with FE65-P2 give significant confidence in the RD53-A design.

\subsection{Monolithic Sensors}

As an alternative to hybrid modules, HV-CMOS pixel detectors are being investigated. Originally designed for charge collection in an epitaxial layer ( $10 \mu \mathrm{m}$ to $20 \mu \mathrm{m}$ thick), new approaches have them coping with the rate and radiation environment expected at the HL-LHC. Options for allowing high depletion voltages and large depletion depths as well as radiation hardness improvements and device thinning are being studied.

Currently the studies on HV-CMOS devices aim for demonstrating the feasibility of its usage in the outer layers of ITk. For this several technologies have been explored and characterised as stand-alone sensors as well as bonded to the FE-I4 pixel chip (as a 'hybrid') either via bump bonds or via glue bonding (capacitively coupled pixel detector, CCPD). The promising results should lead to a large scale demonstrator chip in 2017 showing usability of the CMOS technology in ATLAS ITk.

\section{Powering and Detector Control}

\subsection{Serial Powering}

Powering each of the 10,000 ITk modules in parallel would lead to an unacceptable amount of material inside the detector volume. Therefore, powering options with active voltage regulators inside the detector volume have to be taken into account and as baseline serial powering has been chosen. In this concept a chain of modules is supplied by a constant current source and the modules themselves will perform the current to voltage conversion using built-in shunt regulators in the FE-chips. This way the current being transported is only the current of a single module, while the voltage is the sum of voltages needed by all modules. The modules in the powering chain will operate on different ground levels, so that the communication path has to be AC-coupled.

Tests with serially powered FE-I4 modules, which already include the shunt-regulator foreseen for ITk (an LDO-shunt regulator), show that the power scheme has no impact on the chip operation and functionality. Neither cross-talk nor noise increase could be observed and the minimum tunable threshold is also not influenced by the powering [11].

\subsection{Detector Control}

To ensure safety, reliable control and information for debugging the ITk Pixel a Detector Control System (DCS) [12] is foreseen. It provides three independent control and information paths, which differ in granularity, availability, and reliability.

The safety path, which has the lowest granularity, consists of a hard-wired interlock system, which acts directly on power supplies or other equipment, if the safe operation of the detector cannot be guaranteed any more.

The control and feedback path acts on module level. It is the interface between operator and detector. This path enables steering of all components of the detector and provides monitoring 




Figure 4. Diagram of how a Pixel Serial Powering Protection chip would be integrated into a serial powering chain.

information as feedback. It is made up by the DCS network, consisting of DCS chip and DCS controller, as described in Section 4.2.1.

The diagnostics path is embedded into the module's data path and provides the operator with additional monitoring values in order to debug the behaviour of the detector or tune its performance. It delivers information on read-out chip level and is therefore the path with the finest granularity.

\subsubsection{The DCS Chip and Controller}

The main component of the control path is the DCS chip [13] which provides the monitoring and control capabilities on module level. Figure 4 shows how the DCS chip is integrated within the serial power chain. From the DCS computer the commands are sent to the DCS controller over a long term protocol. The $\mathrm{CAN}^{1}$ protocol is currently envisioned for use.

The DCS chip is specially designed to work in a serial power chain. It is placed on the module flex of each pixel module. It includes bypass capability for the modules, realised by a shunt transistor. The bypass can be activated or deactivated by command. Furthermore, a hardwired automatic activation of the bypass transistor in the case of over-voltage or over-temperature is included. The thresholds for the automatic activation are defined with external components on the flex print. This bypass is off after power-up or in the case of power-loss. For feedback the DCS chip includes an $\mathrm{ADC}$, which allows for monitoring of the module temperature and voltage. As the DCS chip is mounted in close vicinity to the detector modules, even in the innermost layer, it needs to fulfil the same radiation hardness as the read-out chips of the innermost pixel layer. The DCS chips are powered independently from the read-out chips. The DCS chips of one serial input current $\left(I_{S P}\right)$ chain share the same power line (see Figure 4$)$. The power return of the DCS chips is merged with the serial supply current $I_{S P}$. The DCS power line is also used as an external reference for detecting drifts of the internal reference. There are three independent communication lines to the DCS chips of one $I_{S P}$ chain. The DCS chips are AC coupled to the communication lines. Currently up to eight modules are foreseen to build one $I_{S P}$ chain. The DCS controller realises the communication between the DCS computer and the DCS chip. It serves as a bridge between a long range communication from the computer to the short range communication used by the DCS chip.

${ }^{1}$ CAN: Controller Area Network 


\section{Detector Readout Architecture}

Due to the very high radiation levels expected in the innermost detector volume, placing the optoelements at the end of staves or rings is not feasible. Therefore, the data transmission link will be split into an optical part, running from the counting caverns down to the ID-end-plate region (final location still to be defined) and an electrical part, connecting the opto-components to the front-end electronics on the rings or staves. The off-detector components don't need to be radiation hard and therefore can be off-the-shelf commercial components. Optical plug-ins and FPGA based signal processing logic will be placed here. The optical signal will run via optical fibres, which need to be radiation hard to withstand the irradiation along the routing path up to the opto-converter boards. The electrical part needs to serve the data transmission between optical converters and front-end electronics at the needed bandwidth, which depends on the location inside the detector, and must not introduce too much material into the detector not to disturb the particle flight towards the outer subdetectors. As the innermost layers will run at the highest bandwidth, this is the driving factor for the development. Very thin cables serving a bandwidth of around $5 \mathrm{Gbit} / \mathrm{s}$ are under development and study.

The final read-out electronics is still under discussion [14]. As baseline the $\mathrm{TTC}^{2}$ links should operate at a bandwidth of $160 \mathrm{Mbit} / \mathrm{s}$, likely using $8 \mathrm{~b} / 10 \mathrm{~b}$ encoding. This makes sending of more complex commands than a simple trigger signal possible. The TTC links are aggregated using the $\mathrm{GBT}^{3}$ ASIC, which is currently under development at CERN. In contrast, the data links will use the encoding as provided by the read-out chip, such as $64 \mathrm{~b} / 66 \mathrm{~b}$, possibly after multiplexing of the links of read-out chips on one module in case the available bandwidth allows this. The data links will operate at an optical bandwidth of $5 \mathrm{Gbit} / \mathrm{s}$ having lower speed electrical links being aggregated into the faster optical one.

\section{Conclusion}

The development of the components for the ATLAS ITk Pixel detector is ongoing. R\&D activities in the sensor and read-out chip area show already feasible solutions for a future module concept. Alternative approaches such as monolithic pixel chips are also being studied. Simulation studies on the layout options as well as the physics performance will indicate the best layout, the requirements to be put for the electronics and the read-out bandwidths. Intense investigations in meeting the known or assumed requirements for the ITk pixel operation are driving the R\&D effort in all the collaborating labs. The Technical Design Report for the ITk Pixel detector is due by end of 2017. The collaboration is putting high priority on making baseline decisions where several options are still present.

\section{Acknowledgments}

I would like to acknowledge all the colleagues and institutes who contributed to this paper in terms of provided material and working on the achieved results on the various topics discussed.

2TTC: Timing, Trigger, Control

${ }^{3}$ GBT: Gigabit Transceiver 


\section{References}

[1] ATLAS Collaboration, The ATLAS Experiment at the CERN Large Hadron Collider, JINST 3 (2008) S08003

[2] STEP1 ITk Layout Studies.

https://atlas.web.cern.ch/Atlas/GROUPS/PHYSICS/PLOTS/ITK-2016-001/

[3] S. Terzo, Development of radiation hard pixel modules employing planar n-in-p silicon sensors with active edges for the ATLAS detector at HL-LHC, PhD thesis: Technical University of Munich ()2015), https://publications .mppmu .mpg.de/2015/MPP-2015-291/FullText .pdf.

[4] N. Savic et al., Thin n-in-p planar pixel modules for the ATLAS upgrade at HL-LHC, Proceedings of the 14th Vienna Conference on Instrumentation (2016), submitted

[5] N. Savic et al., Investigation of thin n-in-p planar pixel modules for the ATLAS upgrade, Proceedings of the 18th IWORID workshop, submitted to JINST (2016).

[6] J. Lange et al., 3D silicon pixel detectors for the High-Luminosity LHC, Proceedings of the 8th International Workshop on Semiconductor Pixel Detectors for Particles and Imaging, to be published in JINST. September, 2016. arXiv:1610.07480v2

[7] C. J. Kenney, S. Parker and E.Walckiers, Results from 3-D silicon sensors with wall electrodes: near-cell-edge sensitivity measurements as a preview of active-edge sensors, IEEE Transactions on Nuclear Science 48 (2001) 2405, ISSN: 0018-9499.

[8] M. Garcia-Sciveres and J. Christainsen, RD Collaboration Proposal: Development of pixel readout integrated circuits for extreme rate and radiation, CERN-LHCC-2013-008 (2013), URL: http://cds.cern.ch/record/1553467

[9] F. Faccio et al., Radiation-Induced Short Channel (RISCE) and Narrow Channel (RINCE) Effects in 65 and 130 nm MOSFETs, IEEE Trans. Nucl. Sci. 62 (2015) 2933, ISSN: 0018-9499.

[10] T. Heim et al., FE65-P2: a Prototype Pixel Readout Chip in 65nm Technology for HL-LHC Upgrades, to be submitted to 2016 IEEE Nuclear Science Symposium Conference Record (2016).

[11] V. Filimonov, Serial Powering Pixel Stave Prototype for the ATLAS ITk upgrade, Proceedings of TWEPP 2016, to be published in JINST

[12] S. Kersten, L. Püllen, C. Zeitnitz, Ongoing studies for the control system of a serially powered ATLAS pixel detector at the HL-LHC, JINST 11 (2016) C02070

[13] N. Lehmann, M. Karagounis, S. Kersten, C. Zeitnitz, Development of a Detector Control System, JINST 11 (2016) C11004

[14] M. Wensing et al. ATLAS Phase-II-Upgrade Pixel Data Transmission Development, Proceedings of TWEPP 2016, to be published in JINST 